\title{
Evidence for the Role of Proton Shell Closure in Quasifission Reactions from X-Ray Fluorescence of Mass-Identified Fragments
}

\author{
M. Morjean, ${ }^{1,}{ }^{*}$ D. J. Hinde, ${ }^{2}$ C. Simenel, ${ }^{2}$ D. Y. Jeung, ${ }^{2}$ M. Airiau ${ }^{3,4}$ K. J. Cook,${ }^{2}$ M. Dasgupta, ${ }^{2}$ A. Drouart ${ }^{4}$ \\ D. Jacquet, ${ }^{3}$ S. Kalkal, ${ }^{2, \dagger}$ C. S. Palshetkar, ${ }^{2,}$ E. Prasad, ${ }^{2,}$ D. Rafferty, ${ }^{2}$ E. C. Simpson, ${ }^{2}$ \\ L. Tassan-Got, ${ }^{3}$ K. Vo-Phuoc, ${ }^{2}$ and E. Williams ${ }^{2}$ \\ ${ }^{1}$ GANIL, CEA/DRF and CNRS/IN2P3, B.P. 55027, F-14076 Caen Cedex, France \\ ${ }^{2}$ Department of Nuclear Physics, Research School of Physics and Engineering, \\ The Australian National University, ACT 0200, Australia \\ ${ }^{3}$ Institut de Physique Nucléaire, CNRS-IN2P3, Université Paris-Sud, \\ Université Paris-Saclay, F-91406 Orsay Cedex, France \\ ${ }^{4}$ Irfu, CEA, Université Paris-Saclay, F-91191 Gif-sur-Yvette, France \\ (Received 5 July 2017; revised manuscript received 19 October 2017; published 28 November 2017)
}

\begin{abstract}
The atomic numbers and the masses of fragments formed in quasifission reactions are simultaneously measured at scission in ${ }^{48} \mathrm{Ti}+{ }^{238} \mathrm{U}$ reactions at a laboratory energy of $286 \mathrm{MeV}$. The atomic numbers are determined from measured characteristic fluorescence $\mathrm{x}$ rays, whereas the masses are obtained from the emission angles and times of flight of the two emerging fragments. For the first time, thanks to this full identification of the quasifission fragments on a broad angular range, the important role of the proton shell closure at $Z=82$ is evidenced by the associated maximum production yield, a maximum predicted by time-dependent Hartree-Fock calculations. This new experimental approach gives now access to precise studies of the time dependence of the $N / Z$ (neutron over proton ratios of the fragments) evolution in quasifission reactions.
\end{abstract}

DOI: 10.1103/PhysRevLett.119.222502

Since the mid-1970s, it has been known that the formation of superheavy nuclei by fusion is hindered by out-of-equilibrium mechanisms [1-3]. In these mechanisms, the available kinetic energy can be totally dissipated, and large mass transfers between the projectile and the target can occur, leading to emerging fragments quite difficult to distinguish from fragments arising from fusion followed by fission (that might be mass symmetric or asymmetric) [4-7]. Because of these characteristics, the generic name quasifission $(\mathrm{QF})$ is nowadays often used for all these mechanisms. Since the pioneering works, many experimental aspects of QF have been explored [8-17], and dynamical models, macroscopic or microscopic, have been developed in order to reproduce cross sections, distributions of mass, angle, kinetic or excitation energy, and some of the correlations between these observables [15,18-25]. Considering the huge experimental difficulties to extract in a nonarbitrary way small cross sections of fusion followed by fission from dominant quasifission cross sections, a key issue for superheavy nucleus formation studies, it is now essential to get a very good understanding of the QF mechanisms and to confront and improve the models with unambiguous exclusive data in order to reach reliable predictive capacities.

A simultaneous determination of the fragment atomic number $(Z)$ and mass $(A)$ formed in $\mathrm{QF}$ or in fission processes remains nowadays a challenge [26-30], especially difficult because these quantities are most of the time measured after particle evaporation. In this Letter, an experimental approach giving access for QF fragments to $A$ and $Z$ at scission will be presented and the data compared with predictions of a microscopic timedependent Hartree-Fock (TDHF) model [22]. The atomic number was determined from the coincident characteristic fluorescence $\mathrm{x}$ rays, as already attempted for fission fragments [31], whereas the mass was determined from the velocities of the emerging fragments.

$\mathrm{A}^{48} \mathrm{Ti}^{19+}$ beam was accelerated at $5.75 \mathrm{MeV} /$ nucleon by the Australian National University electrostatic accelerator followed by its LINAC postaccelerator. It bombarded $\mathrm{UF}_{4}$ targets highly enriched in ${ }^{238} \mathrm{U}$ on thin carbon or aluminum backings. Because of damage resulting from the beam impact, the targets were rapidly drilled, and different sample thicknesses, ranging from 340 up to $940 \mu \mathrm{g} / \mathrm{cm}^{2}$, have been used during 3 days of data acquisition with a beam intensity $I \approx 12 \mathrm{nA}$. For binary reactions, a very large range of folding angles between the two emerging fragments was covered by two large area position-sensitive multiwire proportional counters $\left(280 \times 360 \mathrm{~mm}^{2}\right)$, MWPC1 and MWPC2. They were positioned on opposite sides of the beam at $d_{1}=195$ and $d_{2}=180 \mathrm{~mm}$ from the target, covering the angular ranges $53^{\circ} \leq \theta_{1} \leq 124^{\circ}$ and $20^{\circ} \leq \theta_{2} \leq 80^{\circ}$, respectively. Coincident photons were detected by three planar germanium detectors $\left(500 \mathrm{~mm}^{2}\right.$, $1 \mathrm{~cm}$ thick each) located at $6 \mathrm{~cm}$ from the target. These detectors were positioned at the same polar angle $\theta=143^{\circ}$ 


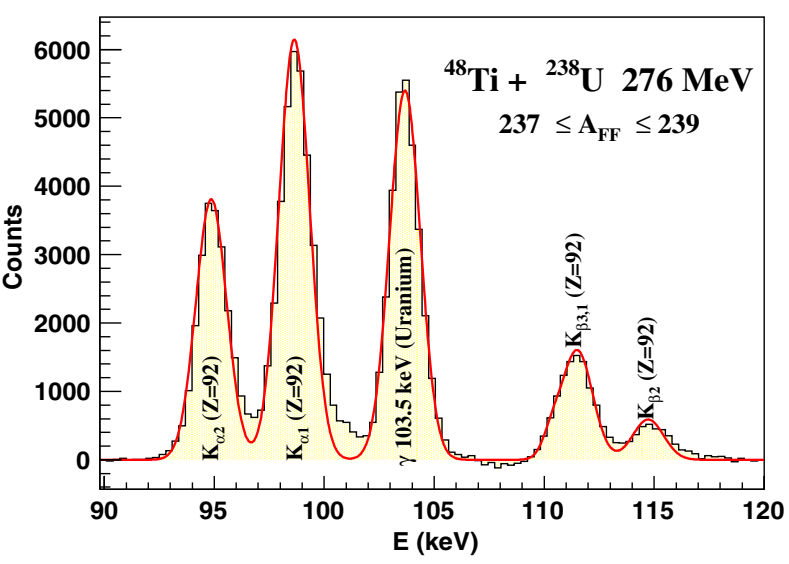

FIG. 1. Photon energy spectrum for fragments with $A=238 \pm 1$. The red line is a fit to the data (see the text).

but at different azimuthal angles $\phi=90^{\circ}, 330^{\circ}$, and $210^{\circ}$ with respect to the plane containing the target and the MWPC centers.

An absolute time calibration could be achieved with a precision better than $200 \mathrm{ps}$ thanks to the kinematical correlation between the detection angles and the velocities for elastically scattered projectile and target nuclei detected on broad angular ranges. A variance $\sigma=2.6 \mathrm{amu}$ has been inferred for the mass resolution from a dedicated measurement at $3.5 \mathrm{MeV} /$ nucleon, an energy much below the Coulomb barrier in which only projectile and target nuclei could be detected. With this resolution, the average masses are quite accurately determined, since, from the Gaussian mass distributions measured at $5.75 \mathrm{MeV} /$ nucleon for elastic and weakly inelastic reactions, the actual projectile and target masses are obtained with a precision better than \pm 1 amu over the whole angular range. For the germanium detectors, an energy resolution $\sigma=400 \mathrm{eV}$ is achieved after a Doppler correction in the whole energy range involved, resulting essentially from the aperture of the germanium detectors that precludes more accurate corrections.

Figure 1 shows the photon energy spectrum for heavy fragments with $A=238 \pm 1$. Uranium characteristic $K \mathrm{x}$ rays and the $\gamma$ ray at $103.5 \mathrm{keV}$ from the uranium $\left(4^{+} \rightarrow 2^{+}\right)$transition can be easily identified. The red line is a fit to the data with six Gaussian distributions. The centroid, variance, and normalization factor of each of the six distributions were free parameters in this fit. The six centroids found by the best fit differ by less than $100 \mathrm{eV}$ from the tabulated energies either for the $\gamma$ transition or for $K \mathrm{x}$ rays from $\mathrm{U}^{1+}$ ions (referred to in the literature as diagram rays) [32]. Furthermore, the yield ratios between the different $K$ transitions agree within less than $5 \%$ with the tabulated ones [32]. Both these energies and yield ratios point out that, for the relatively low ionization states involved for the transiently formed unified atoms as well as for the emerging uranium nuclei, the relative populations on the $L$ and $M$ subshells are not sensitively modified

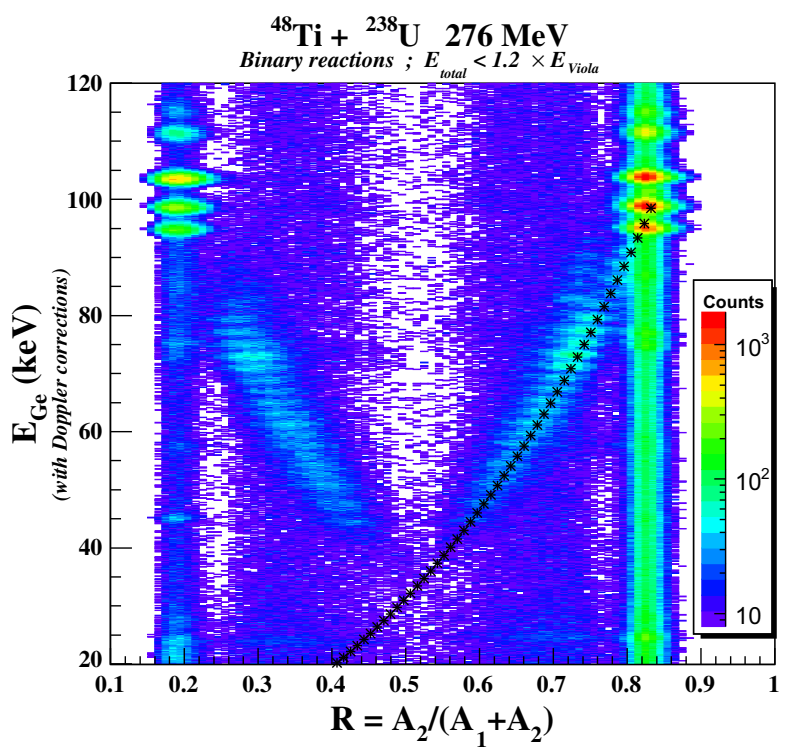

FIG. 2. Photon energy versus mass ratio. The stars indicate the positions of the $K_{\alpha 1}$ X-ray fluorescence lines (see the text).

by the processes responsible for $K$-vacancy creation. Therefore, since on an atomic scale elastic scattering at large angles and very central nuclear reactions correspond to the same impact parameter, the emerging QF fragments should also behave for $K$ x-ray emission like $1^{+}$ions, as already stressed in Ref. [33].

In the following, we shall consider only binary reactions (reactions with only two heavy fragments in the exit channel). Following Ref. [13], binary reactions are selected from the correlation plot between the fragment velocities parallel and perpendicular to the beam axis. In addition, elastic and weakly inelastic reactions are removed by requiring total center-of-mass kinetic energies smaller than $1.2 \times E_{\text {Viola }}, E_{\text {Viola }}$ being the total energy from Ref. [34], adapted for asymmetric fissions. Figure 2 presents the correlation between the photon energy and the mass ratio $R=A_{2} /\left(A_{1}+A_{2}\right)$, where $A_{2}\left(A_{1}\right)$ is the fragment mass in MWPC2 (MWPC1). The picture is not perfectly symmetric with respect to $R=0.5$ due to efficiency losses for folding angles at $R<0.5$. The photon energies have been corrected for the Doppler shift assuming emission from the heaviest of the two fragments, whatever $R$. Despite the selection of inelastic reactions, the different uranium peaks already observed in Fig. 1 are still dominant for mass ratios close to those for the target or projectile nucleus $(R \approx 0.83$ or $0.17)$. For intermediate $R$ values, the Doppler correction reveals lines at constant photon energies. The stars superimposed on Fig. 2 indicate the tabulated $K_{\alpha 1}$ x-ray energies for $Z \leq 92$, positioned at $R$ values corresponding to neutron numbers $N=Z \times\left(N_{T} / Z_{T}\right)$, where the subscript $T$ refers to the target nucleus. The positions of the stars and of the maxima of the photon lines are quite similar, indicating thus that the latter result essentially from $K$ x-ray fluorescence. 


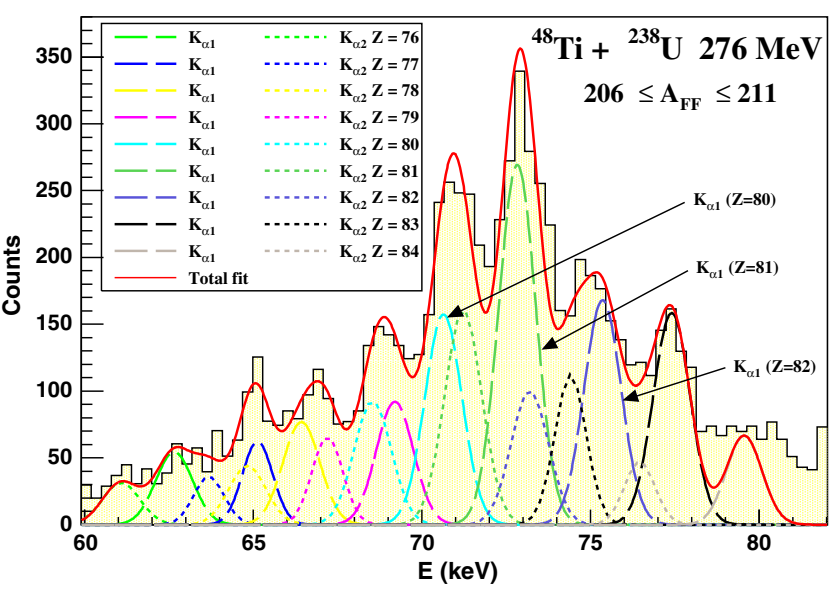

FIG. 3. Doppler-corrected photon energy spectrum (histogram). The full red line is a fit to the data. The different contributions to this fit are shown by dotted and dashed lines (see the text for details).

For the $Z$ range covered in Fig. $2, K_{\alpha 1}(Z)$ and $K_{\alpha 2}(Z+1)$ rays can have energy differences smaller than the experimental resolution and thus cannot be separated. In order to extract the contributions of each element to the lines observed in Fig. 2, photon energy spectra have been constituted for bins of fragment masses, and fits have been performed with a sum of Gaussian distributions representing $K_{\alpha 1}$ and $K_{\alpha 2}$ emission from all possible elements in the energy range considered $\left(K_{\beta}\right.$ lines contribute only weakly to the total spectra and at energies above the $K_{\alpha}$ ones). Figure 3 presents as an example the Doppler-corrected spectrum after background and random coincidence subtractions for fragments with mass $206 \leq A \leq 211(0.72 \leq R<0.74)$. The red solid line shows the best fit, whereas the dashed (dotted) lines correspond for each $Z$ to the $K_{\alpha 1}\left(K_{\alpha 2}\right)$ contributions to this fit. All parameters were allowed to vary freely for fits to mass cuts with $A \geq 190$, whereas, due to lower statistics, the ratios between the $K_{\alpha 1}$ and $K_{\alpha 2}$ yields had to be fixed for $A<190$ at the values for diagram lines in order to reach satisfactory fits. For each $Z$ involved, all the best fits lead for both $K_{\alpha 1}$ and $K_{\alpha 2}$ lines at the tabulated energies $( \pm 0.2 \mathrm{keV})$, with variances $\sigma \approx 400 \mathrm{eV}$. Furthermore, for $A \geq 190$ they lead, within the statistical errors, to $K_{\alpha 1} / K_{\alpha 2}$ ratios in agreement with the ratios for diagram lines [32], as expected from the conclusions of Fig. 1 and from Ref. [33]. Therefore, as exemplified by Fig. 3, the good behavior of all these fits as well in regions where only either a $K_{\alpha 1}$ or a $K_{\alpha 2}$ line is dominant as in regions where they have similar weights provides us with good confidence in the yields inferred for each $Z$.

The $K$ vacancies responsible for $\mathrm{x}$-ray fluorescence from the heavy QF atoms (with a fluorescence yield close to 1) are essentially created during the collision by direct interactions [35] or later by internal conversion processes (IC). Other creation mechanisms like electron shakeoff are much less probable and can be neglected [36]. For QF reactions, the direct vacancies can be created as well in the incoming as in the outgoing part of the interaction, and their probability depends thus slightly on the emerging fragments. Nevertheless, the narrow mass ranges considered in the following imply narrow $Z$ ranges and similar energy distributions, leading to similar $K$-vacancy creation probabilities. Therefore, the total number of direct $K$ vacancies is actually proportional to the number of $K$ vacancies present at scission. The very short $\mathrm{QF}$ lifetime (typically up to $10 \mathrm{zs}$ [13]) does not permit the decay of these vacancies that are all quasiadiabatically transferred to the heavier fragment, as demonstrated by the molecular orbital theory $[35,37]$. The $Z$ distributions at scission can thus be inferred from the characteristic $\mathrm{x}$-ray yields resulting from direct interactions. By contrast, the number of vacancies arising from IC depends strongly on the final isotopes, after neutron evaporation, and their contributions must be subtracted from the measured $\mathrm{x}$-ray yields. IC contributions have been calculated for all the known $\gamma$ - rays in NUDAT2 [38] from all the possible nuclei after evaporation (taking into account the 2.6 amu mass resolution and between zero and four neutrons emitted). Among these rays, the ones not observed (either because they are not emitted or due to too low statistics) have all been assumed to be Gaussian, with a maximum at the background level and $\sigma=400 \mathrm{eV}$, leading to a strong overestimation of their contributions. From the $\gamma$-ray yields and from the $K$ conversion coefficients [39], maximum uncertainties on the $Z$ yields at scission have been determined.

In order to check a possible effect of instrumental electronic nonlinearities on the measured masses, the mass distributions obtained when the heavier QF fragment is detected either in MWPC2 or in MWPC1 have been compared for selections of characteristic $\mathrm{x}$ rays associated with a restricted number of elements. For a given photon selection, provided the electronic chains have good linearities, these two mass distributions must have identical maxima and widths, since the $\mathrm{x}$ rays are emitted by the same ions. Differences of at most $1 \mathrm{amu}$ for the maxima and similar widths have been found in the whole mass range involved, confirming therefore a precision on the mass identification better than $1 \mathrm{amu}$.

Figure 4 presents the yield, the most probable atomic number at scission, and the corresponding neutron number as a function of the fragment mass at scission. The horizontal full lines show the mass range on which the most probable $Z$ have been determined. The latter are determined with good precision in most of the cases for $A \geq 190$, whereas, due to statistics, larger uncertainties are obtained for $A<190$. As expected for $\mathrm{QF}$, the maximum yield is found for weak mass transfers between the target and the projectile, but a secondary maximum is observed at $A=212$ indicated by a dotted vertical line that crosses the data in the middle panel in the vicinity of the proton shell closure $Z=82$. By contrast, it crosses the data in the lower 


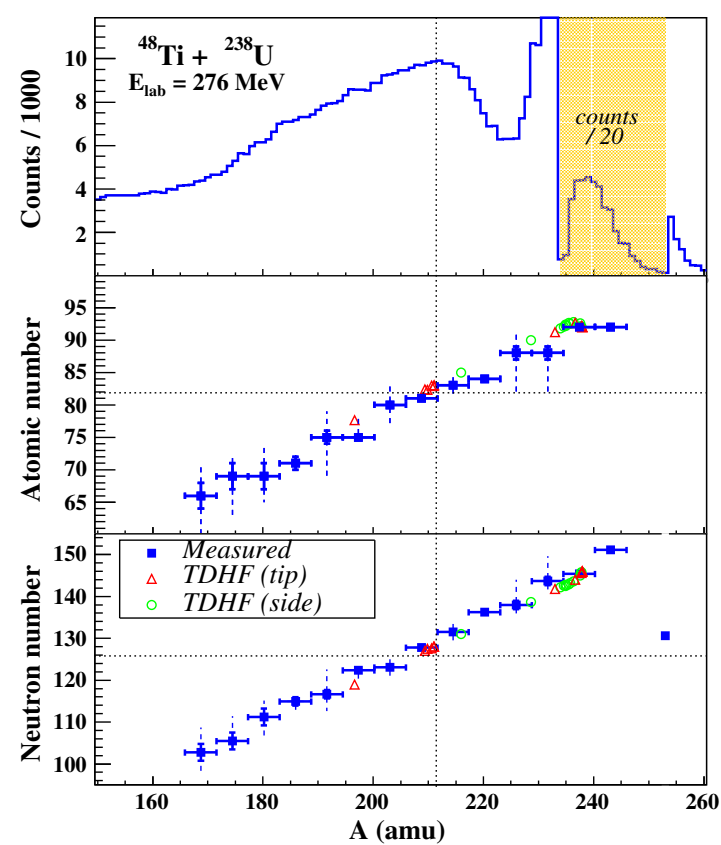

FIG. 4. Yield, most probable atomic number, and neutron number versus mass. The vertical full bars are the statistical errors. The vertical dashed lines show the maximum uncertainties resulting from internal conversion processes (see the text for details).

panel at $N \approx 130$, and the shell closure at $N=126$ would rather correspond to $A \approx 206$, away from the maximum yield even taking into account the statistical uncertainties. Furthermore, on the broad range $200 \lesssim A \lesssim 224$, the influence of the proton shell closure seems to be felt in the middle panel through a lower slope. The strong correlation between the maximum yield and the proton shell closure at $Z=82$ is highlighted by Fig. 5 that presents the $Z$ distribution, as inferred from the fit of the photon energy spectrum between $A=210$ and 214. The statistical errors are represented by the full vertical lines. The dashed lines indicate the maximum uncertainties resulting from IC for $Z=81,82$, and 83 . Because of the very long computational time required to take into account all the possible converted lines, IC contributions have not been calculated for the other $Z$, but, as indicated by vertical arrows, they can only decrease the count numbers and the most probable atomic number associated with the maximum yield is found unambiguously at $Z=82$. If the neutron shell closure played a dominant role, the most probable $Z$ would be between 84 (for $A=210$ ) and 88 (for $A=214$ ). Maximum yields around $A=208$ have been already observed and analyzed $[15,16,40]$ for $\mathrm{QF}$ experiments where only $A$ was measured. They were interpreted as arising either from the $N=126$ neutron shell closure [16] or, according to TDHF calculations that do predict accumulations of fragments with $Z \approx 82$, from the proton shell closure [15]. The simultaneous $A$ and $Z$ measurement provides thus clear evidence for a dominant effect of the closed shell at $Z=82$

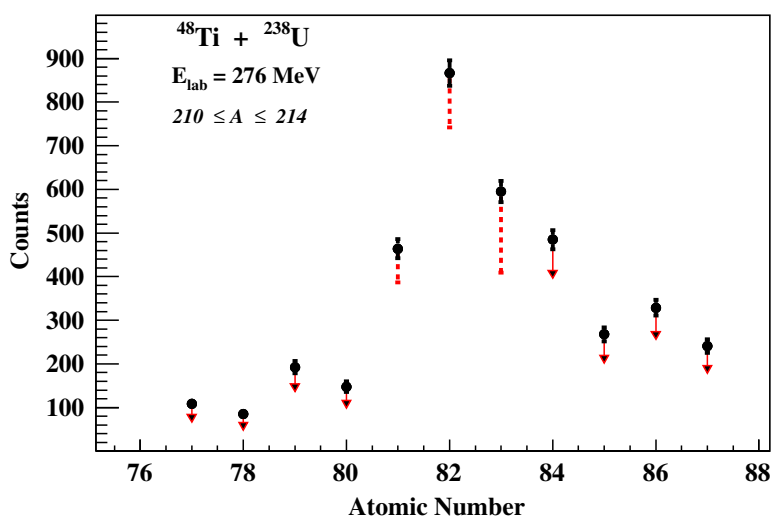

FIG. 5. Atomic number distribution for the quasifission fragments with $210 \leq A \leq 214$ (see the text for details).

on the QF fragment yield. More exclusive experiments are now needed to determine if the maximum yield results from magic number influence during the primary QF fragment formation or from enhanced stability with respect to fission of primary fragments with $Z \approx 82$.

The present experimental approach can be applied to various systems, with or without closed shells, varying the entrance channel mass and charge asymmetries, the projectile kinetic energy, the deformations of the partners, etc. The results should provide for theoretical models realistic controls of the neutron and proton transfers between the partners, leading thus to better predictive powers both for superheavy synthesis by fusion and for new heavy isotope creation by transfers. It must be stressed that such experiments should also provide, besides their contribution in the superheavy domain, quite valuable information on the symmetry energy as used in the nuclear equation of state [41-43].

Triangles and circles in Fig. 4 show the result of TDHF calculations for central collisions for tip and side target orientation (see Supplemental Material [44]) and different energies (simulating for a fixed bombarding energy different angular momenta; see [44]). They confirm important shell effects in the ${ }^{208} \mathrm{~Pb}$ region with the tip orientation. The neutron and proton numbers are relatively well predicted whatever the calculated mass transfers, a quantity that reflects the most probable sticking time [13]. However, the number of protons (neutrons) is always slightly overestimated (underestimated), leading to $N / Z$ values lower than the experimental ones for the heavier QF fragments (and thus higher for the lighter fragments). An overestimation of the $N / Z$ equilibration present in TDHF [45] might explain these differences. A similar behavior with respect to the data is obtained from raw static energy minimizations performed as a function of the mass asymmetry, but the $N / Z$ values for weak mass transfers are slightly lower for the heavy QF fragment than the TDHF ones, most likely due to dynamical effects in TDHF.

The simultaneous measurement of the atomic number and of the mass of quasifission fragments over a broad 
angular range demonstrates the important role played in the final fragment production by the proton shell closure at $Z=82$. Further experiments are now required in order to determine if the dynamical evolution of the composite system in quasifission is sensitive to the shell effects of each of the individual final fragments (as predicted by TDHF microscopic calculations) or if sequential fission processes favor the survival of nuclei with magic proton numbers. The faster $N / Z$ equilibration reached in the calculations might stress an intrinsic limitation to the TDHF approach, possibly due to the symmetry energy (and its density dependence) of the Skyrme energy density used [46]. In the experimental approach followed for the first time here, the uncertainties arising from internal conversion processes can be greatly reduced by reasonable increases of statistics, giving then access to the atomic number distribution for each fragment mass. X-ray fluorescence coupled with accurate mass determination opens thus a broad field of investigations of both quasifission and hot fission processes.

The authors warmly thank N. Lobanov, T. Kibédi, and all the accelerator staff of the ANU Heavy Ion Accelerator for their crucial technical help. The authors acknowledge support from the Australian Research Council through Discovery Grants No. FL110100098, No. FT120100760, No. DP130101569, No. DE140100784, No. DP160 101254, and No. DP170102318. Support for accelerator operations through the NCRIS program is acknowledged. Two of us (C.S. and M. A.) acknowledge support from the Scientific Mobility Program of the Embassy of France in Australia. This research was undertaken with the assistance of resources from the National Computational Infrastructure (NCI), which is supported by the Australian Government.

*morjean@ganil.fr

${ }^{\dagger}$ Present address: Thapar University, Patiala, Punjab 147004, India.

*Present address: Nuclear Physics Division, Bhabha Atomic Research Centre, Mumbai 400085, India.

${ }^{\S}$ Present address: Department of Physics, School of Mathematical and Physical Sciences, Central University of Kerala, Kasaragod 671314, India.

[1] J. Peter, C. Ngo, and B. Tamain, Nucl. Phys. A250, 351 (1975).

[2] R. Bock, Y. Chu, M. Dakowski, A. Gobbi, E. Grosse, A. Olmi, H. Sann, D. Schwalm, U. Lynen, W. Muller, S. Bjornholm, H. Esbensen, W. Wolfli, and E. Morenzoni, Nucl. Phys. A388, 334 (1982).

[3] J. Toke, R. Bock, G. Dai, A. Gobbi, S. Gralla, K. Hildenbrand, J. Kuzminski, W. Muller, A. Olmi, H. Stelzer, B. Back, and S. Bjornholm, Nucl. Phys. A440, 327 (1985).

[4] B. Back, Phys. Rev. C 31, 2104 (1985).

[5] M. Morjean et al., Phys. Rev. Lett. 101, 072701 (2008).

[6] M. O. Frégeau et al., Phys. Rev. Lett. 108, 122701 (2012).
[7] D. Jacquet and M. Morjean, Prog. Part. Nucl. Phys. 63, 155 (2009).

[8] T. Materna et al., Nucl. Phys. A734, 184 (2004).

[9] A. C. Berriman, D. J. Hinde, M. Dasgupta, C. R. Morton, R. D. Butt, and J. O. Newton, Nature (London) 413, 144 (2001).

[10] D. J. Hinde, R. du Rietz, M. Dasgupta, R. G. Thomas, and L. R. Gasques, Phys. Rev. Lett. 101, 092701 (2008).

[11] D. J. Hinde, R. G. Thomas, R. du Rietz, A. Diaz-Torres, M. Dasgupta, M. L. Brown, M. Evers, L. R. Gasques, R. Rafiei, and M. D. Rodriguez, Phys. Rev. Lett. 100, 202701 (2008).

[12] K. Nishio, S. Mitsuoka, I. Nishinaka, H. Makii, Y. Wakabayashi, H. Ikezoe, K. Hirose, T. Ohtsuki, Y. Aritomo, and S. Hofmann, Phys. Rev. C 86, 034608 (2012).

[13] R. du Rietz, E. Williams, D. J. Hinde, M. Dasgupta, M. Evers, C. J. Lin, D. H. Luong, C. Simenel, and A. Wakhle, Phys. Rev. C 88, 054618 (2013).

[14] E. Williams, D. Hinde, M. Dasgupta, R. du Rietz, I. Carter, M. Evers, D. Luong, S. McNeil, D. Rafferty, K. Ramachandran, and A. Wakhle, Phys. Rev. C 88, 034611 (2013).

[15] A. Wakhle, C. Simenel, D. J. Hinde, M. Dasgupta, M. Evers, D. H. Luong, R. du Rietz, and E. Williams, Phys. Rev. Lett. 113, 182502 (2014).

[16] E. M. Kozulin, G. N. Knyazheva, K. V. Novikov, I. M. Itkis, M. G. Itkis, S. N. Dmitriev, Y. T. Oganessian, A. A. Bogachev, N. I. Kozulina, I. Harca, W. H. Trzaska, and T. K. Ghosh, Phys. Rev. C 94, 054613 (2016).

[17] E. Prasad, A. Wakhle, D. J. Hinde, E. Williams, M. Dasgupta, M. Evers, D. H. Luong, G. Mohanto, C. Simenel, and K. Vo-Phuoc, Phys. Rev. C 93, 024607 (2016).

[18] K. Sekizawa and K. Yabana, Phys. Rev. C 93, 054616 (2016).

[19] C. Li, F. Zhang, J. Li, L. Zhu, J. Tian, N. Wang, and F.-S. Zhang, Phys. Rev. C 93, 014618 (2016).

[20] V. E. Oberacker, A. S. Umar, and C. Simenel, Phys. Rev. C 90 , 054605 (2014); A. Umar, V. Oberacker, and C. Simenel, Phys. Rev. C 92, 024621 (2015); A. S. Umar, V. E. Oberacker, and C. Simenel, Phys. Rev. C 94, 024605 (2016).

[21] C. Simenel, D. Hinde, R. du Rietz, M. Dasgupta, M. Evers, C. Lin, D. Luong, and A. Wakhle, Phys. Lett. B 710, 607 (2012).

[22] C. Simenel, Eur. Phys. J. A 48, 152 (2012).

[23] A. Diaz-Torres, G. G. Adamian, N. V. Antonenko, and W. Scheid, Phys. Rev. C 64, 024604 (2001).

[24] V. Zagrebaev and W. Greiner, J. Phys. G 34, 1 (2007).

[25] Y. Aritomo, K. Hagino, K. Nishio, and S. Chiba, Phys. Rev. C 85, 044614 (2012).

[26] T. Enqvist, W. Wlazło, P. Armbruster, J. Benlliure, M. Bernas, A. Boudard, S. Czajkowski, R. Legrain, S. Leray, B. Mustapha, M. Pravikoff, F. Rejmund, K.-H. Schmidt, C. Stéphan, J. Taieb, L. Tassan-Got, and C. Volant, Nucl. Phys. A686, 481 (2001).

[27] P. Armbruster, J. Benlliure, M. Bernas, A. Boudard, E. Casarejos, S. Czajkowski, T. Enqvist, S. Leray, P. Napolitani, J. Pereira, F. Rejmund, M.-V. Ricciardi, K.-H. Schmidt, C. Stéphan, J. Taieb, L. Tassan-Got, and C. Volant, Phys. Rev. Lett. 93, 212701 (2004).

[28] M. Caamaño et al., Phys. Rev. C 88, 024605 (2013).

[29] J.-F. Martin et al., Eur. Phys. J. A 51, 174 (2015). 
[30] A. Chebboubi, G. Kessedjian, H. Faust, A. Blanc, M. Jentschel, U. Köster, T. Materna, O. Méplan, C. Sage, and O. Serot, Nucl. Instrum. Methods Phys. Res., Sect. B 376, 120 (2016).

[31] H. Griffin, J. Radioanal. Nucl. Chem. 142, 279 (1990).

[32] J. Kortright and A. Thomson, in X-Ray Data Booklet, edited by A. Thomson (Lawrence Berkeley National Laboratory, Berkeley, CA, 2009), pp. 1-8.

[33] H. H. Behncke, P. Armbruster, F. Folkmann, S. Hagmann, J. R. Macdonald, and P. H. Mokler, Z. Phys. A 289, 333 (1979).

[34] V. E. Viola, K. Kwiatkowski, and M. Walker, Phys. Rev. C 31, 1550 (1985).

[35] J. Reinhard, W. Greiner, J. Greenbergand, and P. Vincent, in Treatise on Heavy-Ion Science, edited by A. Bromley (Plenum, New York, 1985), Vol. 5.

[36] T. A. Carlson, C. W. Nestor, T. C. Tucker, and F. B. Malik, Phys. Rev. 169, 27 (1968).

[37] R. Anholt, Rev. Mod. Phys. 57, 995 (1985).
[38] NUDAT2, National Nuclear Data Center, information extracted from the NuDat 2 database, http://www.nndc.bnl .gov/nudat $2 /$.

[39] T. Kibédi, T. Burrows, M. Trzhaskovskaya, P. Davidson, and C. Nestor, Jr., Nucl. Instrum. Methods Phys. Res., Sect. A 589, 202 (2008).

[40] Y. Aritomo, Phys. Rev. C 80, 064604 (2009).

[41] L. Shi and P. Danielewicz, Phys. Rev. C 68, 064604 (2003).

[42] L.-W. Chen, C. M. Ko, and B.-A. Li, Phys. Rev. Lett. 94, 032701 (2005).

[43] V. Baran, M. Colonna, M. Di Toro, M. Zielinska-Pfabé, and H. H. Wolter, Phys. Rev. C 72, 064620 (2005).

[44] See Supplemental Material at http://link.aps.org/ supplemental/10.1103/PhysRevLett.119.222502 for TDHF calculations

[45] C. Simenel, P. Chomaz, and G. de France, Phys. Rev. Lett. 86, 2971 (2001); Phys. Rev. C 76, 024609 (2007).

[46] K. Ka-Hae, T. Otsuka, and P. Bonche, J. Phys. G 23, 1267 (1997). 